\title{
Observational review on global waves
}

\author{
D. Banerjee \\ Indian Institute of astrophysics, Koramangala, \\ Bangalore 560034, India \\ email: dipu@iiap.res.in
}

\begin{abstract}
With multi-wavelength observations from ground and space-based instruments it has been possible to detect waves in a number of different wavelengths simultaneously and to, consequently, study their propagation properties. High-resolution wave observations combined with forward MHD modelling can give an unprecedented insight into the connectivity of the magnetized solar atmosphere, which further gives us a realistic chance to construct the structure of the magnetic field in the stellar atmosphere. This type of exploration is also termed as magnetic seismology. In this review I will focus on global waves, like EIT waves. I will also address the possibility of finding out the properties of magnetic structures while studying the interaction of global waves with coronal loops. A Promising new way to probe stellar atmosphere is to use our knowledge of coronal seismology on the Sun and to apply it to more distant stars. It will also enable us to measure properties such as the lengths of loops linked with stellar flares and the strengths of coronal magnetic fields on stars. In the last part I will review the current status of the stellar coronal seismology.
\end{abstract}

\section{Introduction}

One of the earliest observations of global waves known, is that of the chromospheric Moreton waves (Moreton and Ramsey 1960). It was seen in the wings of $\mathrm{H} \alpha$, propagating in the hot chromosphere, with speeds of $400-2000 \mathrm{~km} \mathrm{~s}^{-1}$. Based on their propagation characteristics, Moreton waves are interpreted as fast shock waves. Further unambiguous evidence for large-scale coronal propagating disturbances initiated during the early stages of a flare and/or CME, has been provided by recent EUV Imaging Telescope (EIT) observations onboard SOHO in the $195 \AA$ bandpass. Thompson et al., (1999) reported first on these phenomena based on their SOHO0/EIT observations. Although this instrument has a relatively poor temporal and spatial resolution, there are already more than 200 wavelike events found (Klassen et al. 2000; Biesecker et al. 2002). Since these global waves were first seen by the SOHO/EIT instrument, they were labeled as "EIT waves". EIT waves have circular or arc-shaped fronts of enhanced emission and are generated in or near an active region. Thomspon et al. (1999) showed that the EIT and Moreton waves are quite cospatial when the two waves are observed in a same event.

Then, there is a third kind of global waves, namely X-ray waves as reported by Warmuth et al. (2005). X-ray waves also seem to originate from a flare, and are often cospatial with EIT and Moreton waves. The fact that so many coronal and chromospheric waves are cospatial allows one to conclude that they are part of the same decelerating disturbance. But still there is an ongoing debate about whether the EIT waves are a coronal counterpart of Moreton waves or not. In this review I will present an overview on the subject and express my views on the current state of understanding. 


\section{A Few examples}

Table 1 provides a summary of different observed global waves. An interesting event

Table 1. Overview of the properties of global waves associated with flares.

\begin{tabular}{c|c|l|c|l}
\hline Type of wave & Waveband & Observatory & Locations & References \\
\hline Moreton wave & Ha $\alpha$ & Hida/FMT & Chromospheric & $\begin{array}{l}\text { Discovered by Moreton (1960), Smith \& } \\
\text { Harvey (1971) and identified as the MHD } \\
\text { fast-mode shock wave by Uchida (1968) }\end{array}$ \\
\hline EIT wave & EUV & $\begin{array}{l}\text { SOHO/EIT \& } \\
\text { TRACE }\end{array}$ & coronal & $\begin{array}{l}\text { Thompson et al. (1999), Klassen et al. } \\
\text { (2000); Biesecker et al. (2002); Ballai } \\
\text { et al. (2005) }\end{array}$ \\
\hline X-ray wave & soft X-ray & $\begin{array}{l}\text { Yohkoh/SXT \& } \\
\text { Hinode/XRT }\end{array}$ & coronal & $\begin{array}{l}\text { Khan and Hudson (2000); Khan and } \\
\text { Aurass (2002); Narukage et al. (2002); } \\
\text { Hudson et al. (2003); Narukage et al. } \\
(2004)\end{array}$ \\
\hline
\end{tabular}

was observed on 4 November, 1997 (Eto et al. 2002), at the time of an intense flare (X2.1 in the NOAA/GOES classification). A Moreton wave was observed in $\mathrm{H}-\alpha+0.8 \AA$ and H- $\alpha-0.8 \AA$ with the Flare-Monitoring Telescope (FMT) at the Hida Observatory. At the same time, an EIT wave was observed in EUV with EIT. There is an ongoing debate about whether the EIT waves are a coronal counterpart of Moreton waves or not. EIT and Moreton waves are sufficiently different and some have theorized that they are two entirely different populations, which originate from different sources (Eto et al. 2002). Moreton waves are strongly-defined, narrow, semi-circular fronts, while EIT waves are broad $(\approx 100 \mathrm{Mm})$, extremely diffuse, and usually produce circular wave fronts. Moreton waves have relatively short lifetimes (usually $<$ ten minutes), and have shown cospatial observational signatures between the chromosphere and the soft x-ray corona (Khan and Aurass 2002). EIT waves are primarily visible in the lower corona (at one -two MK), but typically have lifetimes of over an hour and can travel the entire diameter of the Sun while remaining coherent. It appears that there could be two types of wave phenomena in the corona during an eruption, a fast-moving wave which is the coronal counterpart of the $\mathrm{H}-\alpha$ Moreton wave (or the coronal Moreton wave), and a slower moving one which is the EIT wave, with diffuse fronts. SOHO-EIT may catch several wave fronts and at most one front of the coronal Moreton wave in one event if the coronal Moreton wave is moving very fast. We should also point out that although Moreton waves are always viewed in conjunction with EIT waves, the converse is not true, even in high-cadence data.

Okamato et al. (2004) Studied several EIT waves with simultaneous phenomena seen in $\mathrm{H}-\alpha$ in order to address the question of what an EIT wave is. They surveyed the events associated with solar flares larger than GOES M-class in $1999-2002$. The $\mathrm{H}-\alpha$ data were taken with the Flare-monitoring Telescope (FMT) at the Hida Observatory of Kyoto University. They also pointed out that a Moreton wave often triggers a filament oscillation, or a winking filament (Smith \& Ramsey 1964). This is a phenomenon in which a filament disappears and reappears repeatedly at a specific wavelength, since it moves first to the red wing and then to the blue wing when a Moreton wave hits it. It has been reported that even when Moreton waves are not clearly seen, filament oscillations are observed. Hence, the observation of such a winking filament teaches us the existence of an invisible Moreton wave (Smith \& Ramsey 1964; Ramsey \& Smith 1965; Smith \& Harvey 1971; Eto et al. 2002).

An example of the flare associated Moreton wave is shown in Figure 1, as reported 
in Okamato et al. (2004). The flare on 2000 June 15 was classified as an M2.0 GOES flare. The EIT wave was found at 23:47 and 23:59 UT, and the Moreton wave was observed at 23:39 and 23:40 UT. After 23:41 UT, the Moreton wave became invisible. Therefore, the Moreton wave appears in only two frames. Figure 1 shows the FMT images observed in $\mathrm{H}-\alpha+0: 8$ / AA and the relationship between the Moreton wave and the EIT wave. The Moreton wave is shown as intensity images (Fig. 1b) and running-difference images (Fig. 1c) magnified in the box of Figure 1d. Running-difference images show the change between consecutive images by subtracting a previous image from themselves digitally. Figure 1d is a cartoon of the Moreton- and EIT-wave fronts. Okamato et al.
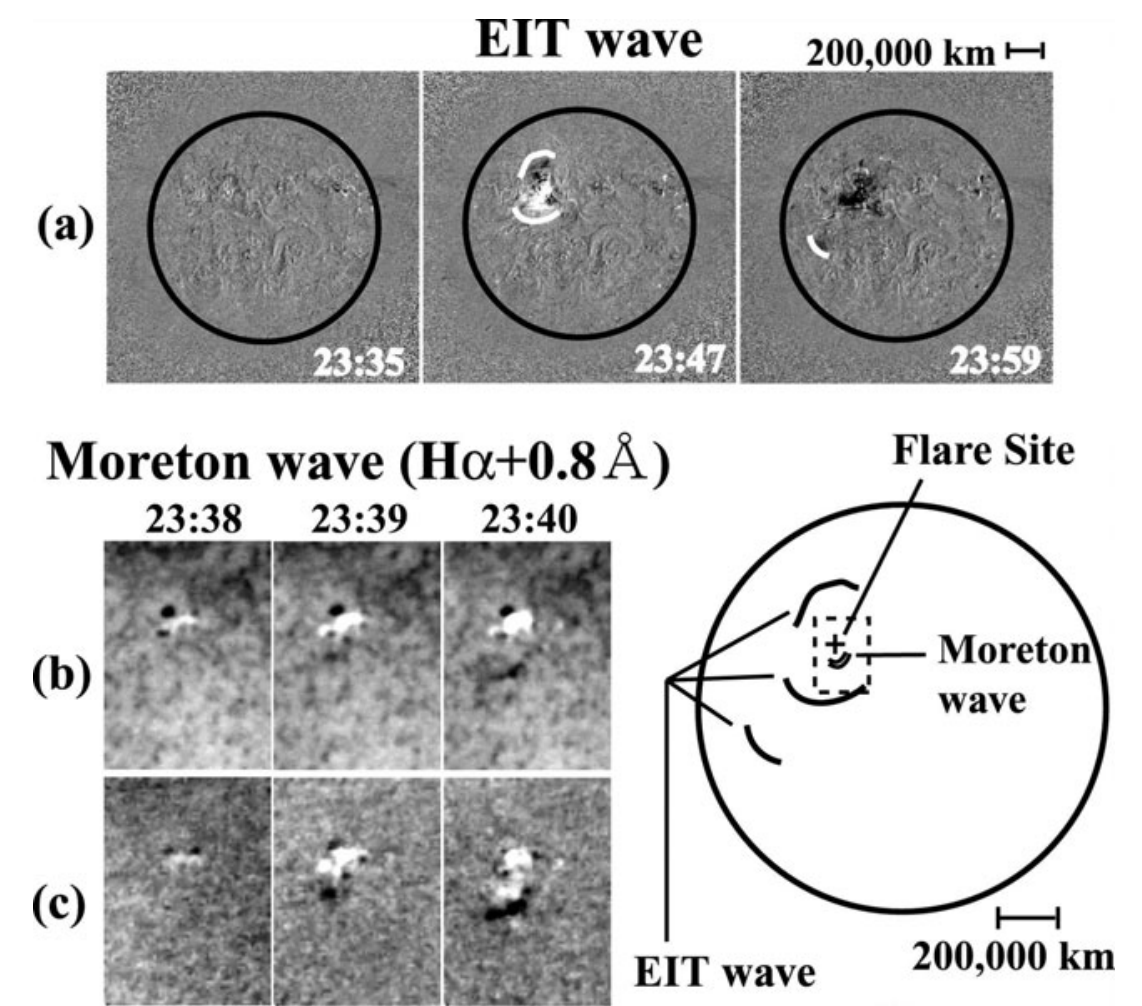

$\longmapsto 50,000 \mathrm{~km}$

(d)

Figure 1. Moreton wave observed in with the Flare-monitoring Telescope (FMT) at Hida Observatory and an EIT wave observed with SOHO EIT at nearly the same time on 2000 June 15. (a) Running-difference images of the EIT wave at 23:35, 23:47, and 23:59 UT. (b) Original images of the Moreton wave at 23:39 and 23:40 UT. (c) Running-difference images of the Moreton wave. (d) Wave fronts of the Moreton wave and the EIT wave. The speed of the Moreton wave and the EIT wave is 397 and $360 \mathrm{~km} \mathrm{~s}-1$, respectively (From Okamato et al. 2004).

(2004) surveyed events in $\mathrm{H}-\alpha$ associated with EIT waves and found three Moreton-wave events and one oscillating filament event. Three out of $14 \mathrm{H}-\alpha$ events associated with large flares, or 21\%, are related to Moreton waves. All these EIT waves are diffuse waves. Thompson et al. (2000) and Warmuth et al. (2001) suggested that diffuse EIT waves are decelerated Moreton waves, while Eto et al. (2002) reported that some EIT waves are not coronal counterparts of Moreton waves. So the point to be note here is that there are cases when both these type of waves has not been observed simultaneously. 


\section{Models of EIT waves}

Opinions are divided on the nature of these global waves between a number of different interpretations (e.g.,, fast magnetohydrodynamic waves, shock waves, non-wave feature, etc.). These global waves originate from impulsive and/or eruptive sources such as flares or coronal mass ejections (CMEs) and are able to travel over very long distances. Sometimes these distances are comparable to the solar radius. It has been proposed, for example, that (i) EIT waves are different entities from Moreton waves, and that (ii) Xray waves as detected by Yohkoh-SXT are, instead, the coronal counterpart of Moreton waves, therefore signifying fast mode MHD waves as predicted by Uchida, Altschuler, and Newkirk (1973). There are also many events in which a sharp EUV wave front is seen to be co-spatial with a soft X-ray (SXR) wave front, the latter exhibiting the characteristics of coronal fast-mode waves (Khan and Aurass 2002). These results tend to favor the coronal fast-mode wave model for EIT waves. Observations show that an EIT wave has two stages: first, there is an early (driven) stage where the wave is correlated with a radio II type burst. This correlation can be attributed to the fact that in the initial stage the propagating wave can excite plasma radiation, accelerate electrons, and create an energized population which serves as the source of the radio emission. The second stage consists of a freely-propagating wavefront. Harra and Sterling (2003) investigated an EIT wave jointly seen by TRACE and CDS/SOHO (JOP70). They concluded that EIT waves consist of a faster propagating, piston-driven portion and a more slowlypropagating portion due to the opening of the field lines associated with an erupting filament. They found that these slowly-propagating waves later interact with coronal loops forcing them to oscillate.

Wills-Davey and Thompson (1999) examined observations that show the first evidence of a coronal wave event seen by TRACE. They concluded that the observed disturbance behaves more like a fast-mode magnetoacoustic wave. Their observations support Uchida's (1968) model of the propagation of an Alfvénic wave in a medium of nonuniform magnetic field. Wills-Davey (2006) has recently developed mapping algorithms that allow automated tracking of a propagating coronal wave, enabling the finding of reproducible fronts and propagation trajectories. The debate on the nature of EIT waves seems to have widened now. While studying the same event simultaneously with different EUV instruments, Wills-Davey, DeForest, and Stenflo (2007) have concluded that fast MHD compressional waves do not properly describe the dynamics of many EIT wave events. The physical properties of EIT waves, their single-pulse, stable morphology, the non-linearity of their density perturbations and the lack of a single representative velocity instead suggest that they may be best explained as a soliton-like phenomena. Given their propagation characteristics and ability to convey information about the medium in which they propagate, global EIT waves, if their mode physics is finally identified properly, could be used as an excellent tool for global coronal seismology.

Ballai, Erdélyi and Pintér (2005) studied TRACE-EUV data to show that these global coronal disturbances are indeed waves with a well-defined period. They showed that EIT waves interact with the coronal loops, and as a result coronal loops begin to oscillate. These induced oscillations are considered to be fast standing kink modes, in good agreement with the theory developed by Roberts, Edwin, and Benz (1984). Ballai, Erdélyi and Pintér (2005) further conjectured that one possible explanation for the different behavior of the same event seen in two wavelengths is that the waves seen in $195 \AA$ (EIT) are just some residuals of a rapid wave propagating in a much denser plasma (e.g., at the chromospheric level in the form of shock waves): these residuals being very similar to a bow wave. The more energetic the wave propagating in the chromosphere is, the larger the 
amplitude that the EIT waves generate. It is possible that small events do not produce large enough waves in the chromosphere to be detected in the low corona. This would explain the relatively small number of EIT waves seen compared to the flaring frequency. Recently Attrill et al. (2007) have claimed that a diffuse EIT wave can be considered

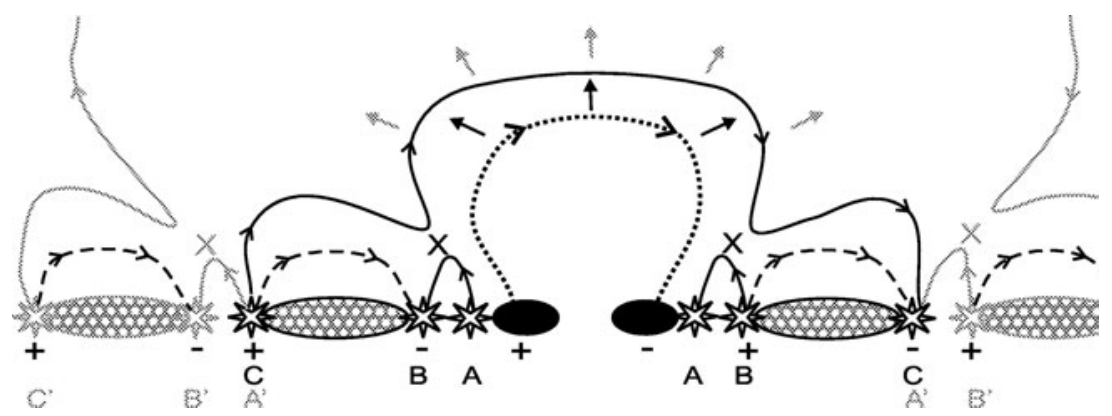

Figure 2. Cartoon illustrating the magnetic reconnection model, as proposed by Attrill et al. 2007, to generate the bright, diffuse coronal wave front, with the observed dual brightenings and two types of dimmings. The expanding CME (dotted line) reconnects with favourably orientated quiet-Sun magnetic loops (dashed lines), displacing the footpoints of the expanding CME (solid line). The crosses mark regions where magnetic reconnection occurs. The dotted/dashed lines show the pre-eruption magnetic structures, the black (gray) solid lines show the result of the first (subsequent) reconnections (From Attrill et al. 2007).

as the magnetic footprint of a CME. They investigated the properties of two classical EIT waves. They showed that the two source regions of the associated CMEs possessed opposite helicities, and the coronal waves display rotations in opposite senses. They observed deep core dimmings near the flare site and also widespread diffuse dimming, accompanying the expansion of the EIT wave, which they attribute to dual brightenings: persistent ones at the outermost edge of the core dimming regions and simultaneously diffuse brightenings constituting the leading edge of the coronal wave, surrounding the expanding diffuse dimmings. They propose a new mechanism where the bright fronts that constitute the diffuse EIT wave front are due to heating caused by the expanding CME magnetic field being thrust into and reconnecting with favorably orientated open or closed magnetic structures. Since the two events they analyzed occurred in a quiet-Sun (QS) environment, they concentrated on interaction with QS loops and scattered open field lines.

In Figure 2, a cartoon (Fig. 4 of Attrill et al. 2007) illustrates how the expanding CME structure (dotted line) reconnects with surrounding favorably orientated QS loops (dashed lines). These reconnections produce brightenings at points $\mathrm{A}, \mathrm{B}$, and $\mathrm{C}$, as a result of chromospheric evaporation (this is a lower energy version of the physics that happens in flares). The flux rope is assumed to expand in all directions at about the same rate and to be anchored in the deep dimming regions. It is the low part of the flux rope (near the footpoints) that is able to reconnect the most with lowlying QS loops. Brightenings A may be mixed with the deep dimming or be spatially unresolvable from brightenings $\mathrm{B}$, thus forming the brightening at the edge of the deep dimmings, while brightenings $\mathrm{C}$ are responsible for the leading edge bright wave front. Together, these brightenings make up the diffuse dual-brightening coronal wave (see Attrill et al. 2007 for further details). 


\subsection{Topological models}

Delannée et al. (2007a) studied an event produced on May 2, 1998. This event consisted of a flare located near AR 8210, a halo CME, dimmings on the solar surface, a Moreton wave and an EIT wave. This event was already studied in 5 articles that concluded that the EIT and Moreton waves are probably magnetosonic waves. Delannée et al. (2007a) noticed that the EIT and Moreton waves present some stationary brightenings remaining at the same location for several minutes and they relate the stationary brightenings to the magnetic field topology. The brightenings in both spectral observations are embedded in the drastic jumps of connectivity of the magnetic field which could be generated by Joule heating or electron acceleration occurring while the magnetic field lines are opening during the CME. They also pointed out that the diffuse arch observed in $\mathrm{H}-\alpha$ is a moving feature and that could not be related to any jumps of connectivity of the magnetic field. So they speculate that a moving diffuse arch could also be observed in EIT if its image time cadence is better.

As a follow up Delannée et al. (2007b) quantitatively addressed this alternative interpretation. Using two independent 3D MHD codes, they performed nondimensional numerical simulations of a slowly rotating magnetic bipole, which progressively resulted in the formation of a twisted magnetic flux tube and its fast expansion, as during a CME. They analysed the origins, the development, and the observability in EUV of the narrow electric currents sheets that appear in the simulations. Both codes gave similar results, which they compared with two well-known SOHO/EIT observations of propagating EIT waves (7 April and 12 May 1997), by scaling the vertical magnetic field components of the simulated bipole to the line of sight magnetic field observed by SOHO/MDI and the sign of helicity to the orientation of the soft X-ray sigmoids observed by Yohkoh/SXT. A large-scale and narrow current shell appears around the twisted flux tube in the dynamic phase of its expansion. This current shell is formed by the return currents of the system, which separate the twisted flux tube from the surrounding fields. It intensifies as the flux tube accelerates and it is co-spatial with weak plasma compression. The current density integrated over the altitude has the shape of an ellipse, which expands and rotates when viewed from above, reproducing the generic properties of propagating EIT waves. The timing, orientation, and location of bright and faint patches observed in the two EIT waves are remarkably well reproduced. They conclude that propagating EIT waves are the observational signature of Joule heating in electric current shells, which separate expanding flux tubes from their surrounding fields during CMEs or plasma compression inside this current shell. They also conjecture that the bright edges of halo CMEs show the plasma compression in these current shells.

\section{Stellar global seismology}

Observations on other stars are rarer. The principal reason for this is that the Sun can be spatially resolved, whereas on stars, the signal for the wave must be strong enough to be observed above the full disk emission. The first oscillation associated with a stellar flare was reported by Rodonó (1974), who observed the flare star II Tau with highspeed optical photometry, and found a long-lived oscillation during flare decay with a mean period of 13s. Andrews (1990) presented optical observations of dMe flare stars, where they observed quasi-periodicities with periods of a few tens of seconds, and put forward the idea that these were coronal loop oscillations. Optical stellar flare oscillations have been observed by Mathioudakis et al. (2003), who found a period of $220 \mathrm{~s}$ in the decay phase of a white-light flare on the RS CVn binary II Peg. Mitra-Kraev et al. 
(2005) presented the first X-ray observation of an oscillation during a stellar flare. The flare occurred on the active M-type dwarf AT Mic and was observed with XMM-Newton. Figure 3 shows the AT Mic light curve. A large flare, starting 15 ks into the observation, increases the count-rate from flare onset to flare peak by a factor of 1.7 , and lasts for $1 \mathrm{~h}$ $25 \mathrm{~min}$. It shows a steep rise (rise time $\tau_{\mathrm{r}}=1300 \mathrm{~s}$ ) and decay (decay time $\tau_{\mathrm{d}}=1700 \mathrm{~s}$ ). There is an extended peak to this flare, which shows clear oscillatory behaviour. Mitra-

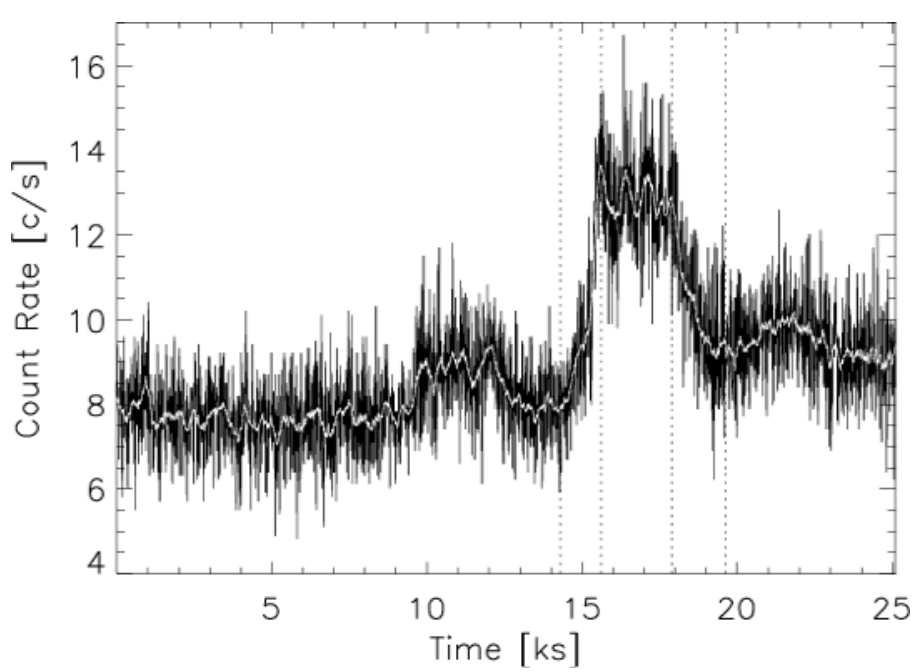

Figure 3. The AT Mic 0.2-12 keV X-ray 10-s bin light curve (shown in black). Overplotted in white is the same light curve smoothed with a sliding time window of $200 \mathrm{~s}$. The time is in ks, starting from the beginning of the observation (2000-10-16 00:42:00). The vertical dotted lines mark the flare start, end of rise phase, end of extended top phase and end of the flare (From Mitra-Kraev et al. 2005)

Kraev et al. (2005) showed that there is an oscillation during the X-ray flare on AT Mic. The oscillation, which has a confidence level $>99.9 \%$, starts when the flare reaches its maximum and continues during the extended flare top. A damped sine curve with an oscillation period of $P=750 \mathrm{~s}$ and an exponential decay time of $\tau=2000 \mathrm{~s}$ fits their data. They interpreted the oscillation as a standing longitudinal slow magneto-acoustic wave tied to the flare loop, with a loop length of $(2.5 \pm 0.2) \times 10^{10} \mathrm{~cm}$. The local magnetic field strength was found to be $105 \pm 50 \mathrm{G}$. Such a flare oscillation provides an excellent opportunity to obtain coronal properties like the size of a flare loop or the local magnetic field strength for the otherwise spatially-unresolved star.

\section{Concluding remarks}

Developing a consistent theoretical understanding of EIT waves is particularly important in the context of new EUV missions. TRACE observations have already shown that wave parameters are easily quantitatively measured with sufficient spatiotemporal resolution (Wills-Davey 2006). If we can correctly model and reproduce EIT waves, we can use wave properties extracted from observations to inverse model the plasma parameters of the affected quiet corona. The higher time cadence of data from Hinode, the Solar Terrestrial Relations Observatory, and the Solar Dynamics Observatory should 
allow a reliable statistical analysis to arrive at better estimates. The multi-wavelength observations, particularly combining spectral information with imaging will be the key for the advancement. Meyer (1968) postulated that large-scale propagating waves can be used for global coronal seismology. However, its successful implementation requires a well-understood theoretical model. Previous studies (Meyer 1968; Ballai et al. 2003) have attempted to calculate quiet Sun magnetic field strengths with global coronal seismology using the assumption that Moreton waves and EIT waves can be modeled as MHD fast-mode waves. Meyer (1968) finds a field strength that appears high, Ballai et al. (2003) finds one that appears low. An accurate wave model may result in a more reasonable field strength calculation, allowing EIT waves to make the transition from coronal phenomenon to observational tool.

\section{References}

Andrews, A. D. 1990, A $\& A, 239,235$

Attrill, G. D. R., Harra, L. K. van Driel-Gesztelyi, L., \& Démoulin, P. 2007, ApJ, 656, L101

Ballai, I., Erdélyi, R., \& Pintér, B. 2005, ApJ, 633, L145

Ballai, I., Thelen, J. C., \& Roberts, B. 2003, A\& A, 404, 701

Biesecker, D.A., Myers, D.C., Thompson, B.J., Hammer, D.M., \& Vourlidas, A. 2002, ApJ, 569, 1009

Delannée, C., Hochedez, J.-F., \& Aulanier, G. 2007a, A\&A, 465, 603

Delannée,C., Török,T., Aulanier, G., \& Hochedez, J.-F. 2007b, Solar Phys., Online first, DOI:10.1007/s11207-007-9085-4

Eto, S. et al. 2002, PASJ, 54, 481

Harra, L.K., \& Sterling, A.C. 2003, ApJ, 587, 429

Hudson, H. S., Khan, J. I., Lemen, J. R., Nitta, N. V., \& Uchida, Y. 2003, Solar Phys., 212, 121

Khan, J. I., \& Hudson, H. S. 2000, Geophysical Research Letters, 27, 1083

Khan, J.I., \& Aurass, H. 2002, AESA, 383, 1018

Klassen, A., Aurass, H., Mann, G., \& Thompson, B.J. 2000, A\&SAS, 141, 357

Mathioudakis, M., Seiradakis, J. H., Williams, D. R., et al. 2003, A\&\&A, 403, 1101

Mitra-Kraev, U., Harra, L.K., Williams, D.R., \& Kraev, E. 2005, A\&SA, 436, 1041

Meyer, F. 1968, in: K. O. Kiepenheuer (ed.) Structure and Development of Solar Active Regions, (Dordrecht: Reidel), IAU Symp. 35, p485

Moreon, G., 1960, AJ, 65, 494

Moreton, G.F., \& Ramsey, H.E. 1960, PASP, 72, 357

Narukage, N., Hudson, H.S., Morimoto, T. et al. 2002, ApJ, 572, L109

Narukage, N., Morimoto, T., Kadota, M. et al., 2004, PASJ, 56, L5

Okamoto et al., 2004, ApJ, 608, 1124

Ramsey, H., \& Smith, S. F. 1965, AJ, 70, 688

Rodonó, M. 1974, A\&A, 32, 337

Roberts, B. Edwin, P. M., \& Benz, A. O. 1984, ApJ, 279, 957

Smith, S. F., \& Ramsey, H. E., 1964, Zeitschrift fr Astrophysik, 60, 1

Smith, S.F, \& Harvey, K.L., 1971, in: C.J. Macris (ed.), Physics of the solar corona,(Dordrecht: Reidel), p.156

Thompson, B.J., Gurman, J.B., Neupert, W.M. et al. 1999, AJ, 517, 151

Thompson, B. J., Reynolds, B., Aurass, H., et al. 2000, Solar Phys., 193, 161

Uchida, Y. 1968, Solar Phys., 4, 30

Uchida, Y., Altschuler, M. D., \& Newkirk, G. 1973, Solar Phys., 28, 49

Warmuth, A. et al. 2001, ApJ, 560, L105

Warmuth, A., Mann, G. \& Aurass, H. 2005, ApJ, 626, L121

Wills-Davey, M. J., \& Thompson, B. J. 1999, Solar Phys., 190, 467

Wills-Davey, M. J. 2006, ApJ, 645, 757

Wills-Davey, M. J., DeForest, C. E., \& Stenflo, J. O. 2007, ApJ, 664, 556 